\title{
Ruptured pyogenic liver abscess in pregnancy: a case report
}

\author{
Smruti Gedam ${ }^{1}$, Shuchita Mundle ${ }^{2 *}$
}

\begin{abstract}
${ }^{1}$ Department of Obstetrics and Gynecology, Government Medical College and Hospital, Nagpur, Maharashtra, India
\end{abstract} ${ }^{2}$ Department of Obstetrics and Gynecology, All India Institute of Medical Sciences, Nagpur, Maharashtra, India

Received: 18 June 2020

Accepted: 10 July 2020

\section{*Correspondence:}

Dr. Shuchita Mundle,

E-mail: drshuchitamundle@rediffmail.com

Copyright: ( ) the author(s), publisher and licensee Medip Academy. This is an open-access article distributed under the terms of the Creative Commons Attribution Non-Commercial License, which permits unrestricted non-commercial use, distribution, and reproduction in any medium, provided the original work is properly cited.

\begin{abstract}
Pyogenic liver abscess during pregnancy is an extremely dangerous condition, both from general as well as obstetric point of view. An interesting case of 24-year primigravida reported at 28 weeks gestation with high grade fever and chills with features of peritonitis is being presented here, which on investigations subsequently was diagnosed to be a case of ruptured pyogenic liver abscess. The case was managed successfully with higher antibiotics and percutaneous drainage of the abscess. The obstetric outcome was successful as well, with spontaneous preterm delivery of 920 gm male child which cried immediately after birth and was managed with appropriate care in premature baby unit.
\end{abstract}

Keywords: Case report, Pregnancy, Pyogenic liver abscess, Ruptured liver abscess

\section{INTRODUCTION}

Pyogenic liver abscess (PLA) complicating pregnancy is extremely rare. ${ }^{1}$ The most common microorganisms reported with this complication are Escherichia coli and Bacteroides spp. and polymicrobial infections. ${ }^{2}$ High index of suspicion, early diagnosis and treatment is key for adequate management and successful outcome; else the consequences could be grave including death. In this report, authors present an interesting case of pregnant women in her late second trimester with complaint of high-grade fever and pain in abdomen, who later on developed signs of peritonitis as a result of ruptured pyogenic liver abscess.

\section{CASE REPORT}

A 24-year-old, primigravida of 28 weeks of gestation (with history of primary infertility since 4 years) reported to emergency department of the study hospital as a referred case from private hospital at nearby sub-district place. From her medical history it was understood that she had 10 days history of high-grade fever associated with chills and rigors, for which patient consulted and got admitted at a private hospital. She was diagnosed as having sepsis (? cause) and received intravenous antibiotics (cefotaxime) for 7 days. When there was no relief in spite of intravenous antibiotics, she was referred to authors hospital. The fever was followed by pain and distension of abdomen in the intervening 5 days.

Thorough examination was conducted on admission. She was conscious, febrile with temp of 102-degree F, pulse rate of $148 / \mathrm{min}$, blood pressure $110 / 70 \mathrm{mmHg}$, tachypneic with respiratory rate of $36 / \mathrm{min}$, shallow breathing with nasal flaring, face was flushed, pallor present and no icterus. Air entry was decreased on right side of chest on respiratory system examination. On abdominal examination, abdomen was distended with gross abdominal wall edema. Guarding and tenderness were significantly evident, more so in the right hypochondrium. Local temperature of abdomen was raised. Uterine height could not be assessed properly, FHS were localized on hand Doppler and the rate was 152 beats per minute. Locally, minimal vulvar edema was evident. On pelvic examination cervical os was closed and there was no evidence of vaginitis. Rest of the systemic examination was within normal limits. After 
primary stabilization, intravenous antibiotics were started and basic blood investigations were ordered.

Ultrasonography (USG) of abdomen done one day prior to admission at study hospital was suggestive of single live intrauterine gestation of 27 weeks and 2 days in cephalic presentation, posterior grade I placenta, adequate liquor and evidence of mild edema around fetal scalp, thorax and abdomen. Hepatomegaly of $19.8 \mathrm{~cm}$ and raised echotexture of liver, gall bladder wall edema and mild ascites were also reported. Routine haematological investigations revealed significant anemia $(\mathrm{Hb}$. -7.6 gm\%), borderline neutrophilic leukocytosis (TLC$11000 / \mathrm{cmm}, \mathrm{N}-78 \%$ ). The liver function and renal function parameters along with serum electrolytes were normal. Serology reports were run for HIV, HBsAg, HCV and VDRL; which were negative. The fever profile, vaginal swab, blood culture was all negative as well. USG was repeated after admission on day two and following observations were noted: liver mildly enlarged $(16.5 \mathrm{~cm})$, heterogeneous hypoechoic collection in liver of size $5.6 \times 6.6 \times 6.5 \mathrm{~cm}(120 \mathrm{cc})$ approx. in right lobe of liver (segment V, VI, VII), with mild ascites with findings suggestive of (?) abscess rupture. Obstetric scan was corresponding to 27 weeks and 6 days. Review USG abdomen from senior radiologist was suggestive of collection of lesser volume $(\sim 90-100 \mathrm{cc})$ in right lobe of liver (segment V, VI, VII) with possible rent in the wall, minimal peri-hepatic collection, mild ascites with largest pocket in right hypochondrium $(40-50 \mathrm{cc})$ and in left hypochondrium $(30-40 \mathrm{cc})$, with right sided pleural effusion. X-ray chest with upper abdomen was suggestive of right sided moderate pleural effusion which was also corroborative of above findings (Figure 1).

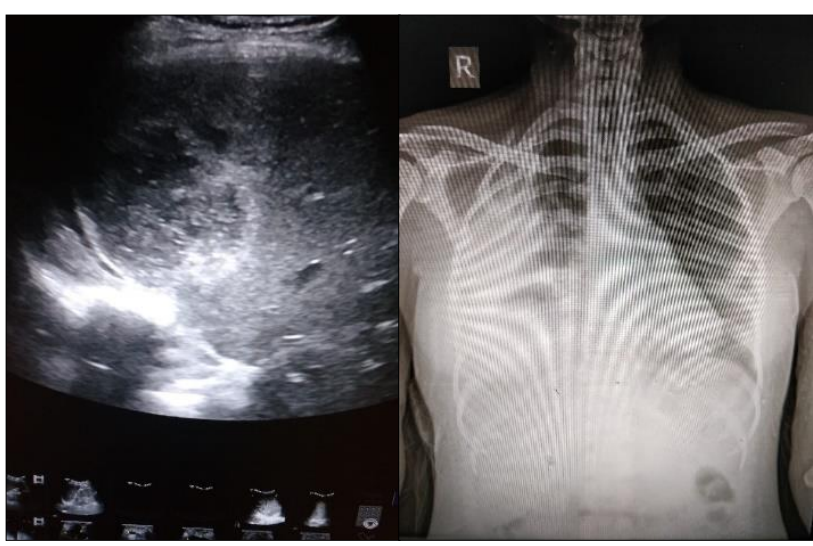

Figure 1: Ultrasound abdomen and $X$-ray chest with upper abdomen (day 2 of admission).

The diagnosis of ruptured liver abscess with peritonitis was arrived. The patient was initially managed conservatively with higher intravenous antibiotics (piperacillin + tazobactum and metronidazole) antipyretics, analgesics and intravenous fluids. One-pint whole blood transfusion was given in view of prevailing anaemia. USG guided ascitic fluid tapping revealed frank pus in peritoneal cavity. Initial surgeon's opinion was in favor of need of urgent exploratory laparotomy in view of ruptured liver abscess with peritonitis. But a multidisciplinary review was sought and decision to continue conservative management with higher antibiotic coverage was taken for the moment keeping comprehensive picture in mind including obstetric outcome, with continued monitoring and scope for SOS review. USG guided percutaneous aspiration of liver abscess was done followed by pigtail catheter insertion. The purulent aspirate was sent for routine microscopy and culture/sensitivity and the sample was positive for plenty of pus cell with no organism grown. USG guided pleural fluid tapping was done in view of right sided moderate pleural effusion. The plural fluid examination report revealed 7-8 RBCs/HPF, 12-14 leucocytes/HPF, neutrophilic leukocytosis (TLC -20000/cmm, N-95\%), low sugar with high protein and no organism grown.

On day six of admission, patient went into spontaneous preterm labor and delivered a male baby weighing 920 gm. Baby cried immediately after birth and was shifted to PBU for further management. Intra and immediate postpartum period was uneventful. Post-delivery review USG abdomen was showing hepatomegaly $(19.6 \mathrm{~cm}$ span), with ruptured liver abscess of increased size of $7.8 \times 8.3 \times 8.5 \mathrm{~cm}(210 \mathrm{cc})$ in segments V, VI, VII. The patient had icterus and liver enzymes were slightly raised. The patient then started responding to the treatment, albeit slowly. On day 20 post admission, the USG abdomen was suggestive of residual collection of $\sim 10 \mathrm{cc}$, semisolid in consistency. Pigtail catheter was then removed. On day 25 review USG was showing $\sim 25 \mathrm{cc}$ liver abscess and all the hematological investigations were normal. The patient was discharged on antibiotics (Tablet levofloxacin for 14 days) and supportive treatment. At one-month follow-up, patient was absolutely asymptomatic with normal abdominal sonography, indicative of complete recovery.

\section{DISCUSSION}

Pyogenic liver abscess (PLA) is a serious, life threatening condition that is difficult to diagnose and treat. Usually the diagnosis is not easy to arrive at, unless there are reasons to suspect the same. The incidence varies from region to region but has been reported to be up to 11 cases/million persons/year on the higher side. ${ }^{3-6}$

Although fever and pain in upper abdomen are the most frequent clinical symptoms, the clinical presentation may vary, may be nonspecific in many cases and hence difficult to diagnose. Most common organisms reported to be associated with this condition are E.coli and Bacteroides spp, with gastro-intestinal track being the usual source of infection. ${ }^{2,7}$ Any degree of mucosal defects, especially in susceptible hosts, through hematogenous spread is the route for the bacteria to gain access to liver through portal circulation. ${ }^{8}$ Similar to clinical findings, laboratory tests are also non-specific in such cases. Most frequent findings are leukocytosis, 
raised ALP, and increased fibrinogen which are not specific during pregnancy. Majority of the cases are diagnosed on ultrasonography of abdomen, the sensitivity of which is reported to be $85.8 \%{ }^{9}$

PLA during pregnancy is an extremely rare entity and poses diagnostic and therapeutic challenges. Being nonspecific in presentation and lab findings, misdiagnosis as well as delayed diagnosis is quite possible. Early treatment initiation is important to avoid potential risk of preterm birth, fetal infection, increased perinatal mortality, multi-organ dysfunction, sepsis, septic shock and even maternal mortality. ${ }^{10}$ Sepsis and septic shock during pregnancy can be fatal, whatever the source is, and the initial stabilization, fluid resuscitation and prompt initiation of broad spectrum antibiotics is very important. Simultaneous efforts must be directed to find out the source of infection (genito-urinary, respiratory, gastrointestinal etc.), which is to be addressed on priority. Like in the present case, the pus needed to be drained along with prompt initiation of appropriate antibiotic. From obstetric point of view, premature or prolonged rupture of membranes and chorioamnionitis should be ruled out and proper history and vaginal examination is vital for the same; as it can be a common source of infection, especially during late pregnancy. ${ }^{10}$ In this case, features of peritonitis guided us for early diagnosis and timely management.

The management of pyogenic liver abscess in pregnancy is tricky, as the general as well obstetric risk needs to weighed in before proceeding with any intervention. A pregnant woman with PLA and poor prognostic factors ideally requires laparotomy in late third trimester. For all those abscesses which are not able to be drained during laparotomy or the patient is unfit to undergo laparotomy during late pregnancy, ultrasound guided drainage in combination with wide spectrum antibiotic coverage is the method of choice. In the first, second and early third trimester, ultrasound guided drainage may be performed as the first line of management, with the patient being closely followed up; and the same was exercised in the present case after multidisciplinary deliberations.

\section{CONCLUSION}

In conclusion, the present case demonstrates that making an accurate and timely diagnosis and adopting multidisciplinary approach to management contributes to a successful clinical outcome in case of pyogenic liver abscess in pregnancy. Although rare, in situation of sepsis or septic shock in pregnancy, apart from common causes of infection, a possibility of liver abscess should be kept in mind and ultrasonographic evaluation of the region should be considered.

Funding: No funding sources

Conflict of interest: None declared

Ethical approval: Not required

\section{REFERENCES}

1. Lindgren P, Pla JC, Hogberg U, Tarnvik A. Listeria monocytogenes-induced liver abscess in pregnancy. Acta Obstet Gynecol Scandinavica. 1997;76(5):4868.

2. Pérez JA, González JJ, Baldonedo RF, Sanz L, Carreño G, Junco A, et al. Clinical course, treatment, and multivariate analysis of risk factors for pyogenic liver abscess. The Am J Surg. 2001;181(2):177-86.

3. Tsai FC, Huang YT, Chang LY, Wang JT. Pyogenic liver abscess as endemic disease, Taiwan. Emerg Infect Dis. 2008;14(10):1592.

4. Meddings L, Myers RP, Hubbard J, Shaheen AA, Laupland KB, Dixon E, et al. A population-based study of pyogenic liver abscesses in the United States: incidence, mortality, and temporal trends. Am J Gastroenterol. 2010;105(1):117-24.

5. Jepsen P, Vilstrup H, Schønheyder HC, Sørensen HT. A nationwide study of the incidence and 30-day mortality rate of pyogenic liver abscess in Denmark, 1977-2002. Aliment Pharmacol Therap. 2005;21(10):1185-8.

6. Kaplan GG, Gregson DB, Laupland KB. Populationbased study of the epidemiology of and the risk factors for pyogenic liver abscess. Clin Gastroenterol Hepatol. 2004;2(11):1032-8.

7. Johannsen EC, Sifri CD, Madoff LC. Pyogenic liver abscesses. Infect Dis Clin North Am. 2000;14(3):547-63.

8. Butler TJ, McCarthy CF. Pyogenic liver abscess. Gut. 1969;10(5):389.

9. Lin AC, Yeh DY, Hsu YH, Wu CC, Chang H, Jang TN, Huang CH. Diagnosis of pyogenic liver abscess by abdominal ultrasonography in the emergency department. Emerg Med J. 2009;26(4):273-5.

10. Kopernik G, Mazor M, Leiberman JR, Barki Y, Hirsch M. Pyogenic liver abscess in pregnancy. Israel J Med Sci. 1988;24(4-5):245-6.

Cite this article as: Gedam S, Mundle S. Ruptured pyogenic liver abscess in pregnancy: a case report. Int J Reprod Contracept Obstet Gynecol 2020;9:3509-11. 